\title{
Characteristics of Missed Synchronous Gastric Epithelial Neoplasms
}

\author{
Bong Eun Lee \\ Department of Internal Medicine, Pusan National University School of Medicine and Biomedical Research Institute, Pusan National \\ University Hospital, Busan, Korea
}

See "Characteristics of Missed Simultaneous Gastric Lesions Based on Double-Check Analysis of the Endoscopic Image" by Eun Jeong Gong, Jeong Hoon Lee, Kyoungwon Jung, et al., on page 261-269.

Endoscopic resection is now established as a standard treatment for gastric epithelial neoplasms such as early gastric cancer and adenoma. Careful preprocedural evaluation and proper patient selection are important for successful therapeutic outcomes; therefore, repeated endoscopy is often planned when a patient is referred, and a more detailed examination is thought to be necessary for deciding the treatment strategy. The development of gastric epithelial neoplasm is associated with atrophy or intestinal metaplasia of background mucosa; thus, synchronous multiple lesions are common. The prevalence of synchronous gastric cancer has been reported to range from $4.8 \%$ to $20.9 \%,{ }^{1-7}$ and the prevalence of synchronous lesions after endoscopic resection has been reported to be $2.0 \%$ to $11.6 \% .^{8-10}$ Missing synchronous lesions may lead to increased cost for additional procedures or even make the initial endoscopic treatment meaningless, especially when the missed lesion is located more proximally, requiring gastrectomy. Therefore, meticulous endoscopic examination so as not to miss synchronous lesions is critical. Endoscopists sometimes perform repeated endoscopy before endoscopic resection not only to evaluate the confirmed lesion but also to

Received: April 14, 2017 Revised: May 12, 2017

Accepted: May 16, 2017

Correspondence: Bong Eun Lee

Department of Internal Medicine, Pusan National University School of Medicine and Biomedical Research Institute, Pusan National University Hospital, 179 Gudeok-ro, Seo-gu, Busan 49241, Korea

Tel: +82-51-240-7869, Fax: +82-51-244-8180, E-mail: bongsul@daum.net

(c) This is an Open Access article distributed under the terms of the Creative Commons Attribution Non-Commercial License (http://creativecommons.org/ licenses/by-nc/3.0) which permits unrestricted non-commercial use, distribution, and reproduction in any medium, provided the original work is properly cited. detect synchronous lesions missed at the initial endoscopy.

Synchronous multiple gastric carcinomas are known to be associated with older age and the degree of intestinal metaplasia in the background mucosa. ${ }^{11}$ Studies have evaluated the features and risks of missed synchronous gastric lesions. Small lesion size, location of the lesion (body), and pathological diagnosis of the primary lesion (adenoma) were reported as risk factors. ${ }^{8,912-15}$ Although the optimal duration of endoscopic examination is still indefinite, the probability of detecting synchronous neoplasms was found to decrease by $6.9 \%$ as the duration of the examination decreased by 1 minute, and procedures that lasted more than 10 minutes detected synchronous lesions more frequently. ${ }^{9}$

In this issue of Clinical Endoscopy, Gong et al. ${ }^{16}$ reported the characteristics of missed gastric synchronous lesions based on the endoscopic images at the referring center, which were compared with images of repeated endoscopy before endoscopic resection. They evaluated 140 patients, of whom 12 (8.6\%) showed 13 synchronous lesions, comprising 10 dysplasias $(76.9 \%)$ and 3 adenocarcinomas (23.1\%). Most synchronous lesions were located in the lower third of the stomach, and the median tumor size was $18 \mathrm{~mm}$. When classifying these synchronous lesions into 3 groups based on a review of referred endoscopic images (group 1, no images of the location of simultaneous lesions; group 2, no corresponding lesion evident in the previous images; and group 3, simultaneous lesions found in the earlier images but without a biopsy), 7 lesions (53.8\%) were classified as group 3, $5(38.5 \%)$ as group 1 , and the remaining (7.7\%) as group 2. Meanwhile, no significant differences were found in the patients' and lesions' 
characteristics and procedure-related factors, including the procedure time in detecting synchronous lesions. Regarding the study results, the authors concluded that lesions disregarded or missed during endoscopy were the main reason for missing synchronous lesions and emphasized the importance of a meticulous and complete endoscopic evaluation to avoid overlooking gastric epithelial neoplasms. Although some limitations were already mentioned in their article and the conclusions from the study are not surprising, which seems almost natural, they presented evidence-based conclusions by analyzing their own experiences.

Missing synchronous gastric lesions can be attributed mainly to poor endoscopic skills or image qualities. However, this is not the only matter of concern for the initial endoscopist or referring center. The abnormality might be subtle and hardly noticeable at the time of initial examination. In addition, atrophic or metaplastic changes of the surrounding mucosa make the detection of the lesions difficult, especially when the lesion does not show apparent surface irregularities or color changes. Furthermore, the appearance of lesions constantly changes, even during the same examination. In these conditions where lesions are difficult to detect, careful observation with appropriate air insufflation and suction to reduce the possibility of missed lesions is the only option. In conclusion, as synchronous gastric lesions can be missed occasionally, even by expert endoscopists, meticulous examination of the entire stomach and awareness of potential blind spots is mandatory during the full endoscopic period. Repeated imaging of suspicious lesions with appropriate air control can help detect subtle changes.

\section{Conflicts of Interest}

The author has no financial conflicts of interest.

\section{REFERENCES}

1. Moertel CG, Bargen JA, Soule EH. Multiple gastric cancers; review of the literature and study of 42 cases. Gastroenterology 1957;32:1095-1103.

2. Kosaka T, Miwa K, Yonemura Y, et al. A clinicopathologic study on multiple gastric cancers with special reference to distal gastrectomy. Cancer 1990;65:2602-2605.

3. Esaki Y, Hirokawa K, Yamashiro M. Multiple gastric cancers in the aged with special reference to intramucosal cancers. Cancer 1987;59:560-565.

4. Kodera Y, Yamamura Y, Torii A, et al. Incidence, diagnosis and significance of multiple gastric cancer. Br J Surg 1995;82:1540-1543.

5. Iwafuchi M, Watanabe $\mathrm{H}$. [Synchronous multiple early gastric cancer; study of surgically resected stomach]. Nihon Rinsho 1996;54:1217-1223.

6. Mitsudomi T, Watanabe A, Matsusaka T, Fujinaga Y, Fuchigami T, Iwashita A. A clinicopathological study of synchronous multiple gastric cancer. Br J Surg 1989;76:237-240.

7. Honmyo U, Misumi A, Murakami A, Haga Y, Akagi M. Clinicopathological analysis of synchronous multiple gastric carcinoma. Eur J Surg Oncol 1989;15:316-321.

8. Kim HH, Cho EJ, Noh E, et al. Missed synchronous gastric neoplasm with endoscopic submucosal dissection for gastric neoplasm: experience in our hospital. Dig Endosc 2013;25:32-38.

9. Kim HH, Kim JH, Kim GH, Choi MG, Jee SR, Song GA. Causes of missed synchronous gastric epithelial neoplasms with endoscopic submucosal dissection: a multicenter study. Scand J Gastroenterol 2013;48:1339-1346

10. Yoo JH, Shin SJ, Lee KM, et al. How can we predict the presence of missed synchronous lesions after endoscopic submucosal dissection for early gastric cancers or gastric adenomas? J Clin Gastroenterol 2013;47:e17-e22.

11. Nitta T, Egashira Y, Akutagawa H, et al. Study of clinicopathological factors associated with the occurrence of synchronous multiple gastric carcinomas. Gastric Cancer 2009;12:23-30.

12. Lee HL, Eun CS, Lee OY, et al. When do we miss synchronous gastric neoplasms with endoscopy? Gastrointest Endosc 2010;71:1159-1165.

13. Sung IK, Kim YC, Yun JW, et al. Characteristics of advanced gastric cancer undetected on gastroscopy. Korean J Gastroenterol 2011;57:288293.

14. Ren W, Yu J, Zhang ZM, Song YK, Li YH, Wang L. Missed diagnosis of early gastric cancer or high-grade intraepithelial neoplasia. World J Gastroenterol 2013;19:2092-2096.

15. Pimenta-Melo AR, Monteiro-Soares M, Libânio D, Dinis-Ribeiro M. Missing rate for gastric cancer during upper gastrointestinal endoscopy: a systematic review and meta-analysis. Eur J Gastroenterol Hepatol 2016;28:1041-1049.

16. Gong EJ, Lee JH, Jung K, et al. Characteristics of missed simultaneous gastric lesions based on double-check analysis of the endoscopic image. Clin Endosc 2017;50:261-269. 